\title{
Elliptic problems with nonhomogeneous boundary condition and derivatives of nonlinear terms
}

\section{Dumitru Motreanu' ${ }^{1}$ and Viorica $\vee$ Motreanu ${ }^{2 *}$}

\section{"Correspondence:}

motreanu@post.bgu.ac.il

${ }^{2}$ Department of Mathematics, Ben

Gurion University of the Negev,

Be'er Sheva, 84105, Israel

Full list of author information is

available at the end of the article

\begin{abstract}
The paper presents existence results for nonlinear elliptic problems under a nonhomogeneous Dirichlet boundary condition. The considered elliptic equations exhibit nonlinearities containing derivatives of the solution.
\end{abstract}

MSC: $35 \mathrm{H} 30 ; 35 \mathrm{~A} 16$

Keywords: quasilinear elliptic problem; nonhomogeneous Dirichlet boundary conditions; existence result

\section{Introduction}

The aim of the paper is two-fold: first, to study nonlinear elliptic problems under nonhomogeneous Dirichlet boundary condition; second, to incorporate in the problem statement nonlinearities exhibiting derivatives of the solution. These requirements need to develop a nonstandard approach, in particular prevent the use of variational methods.

Specifically, we study two problems on a bounded domain $\Omega \subset \mathbb{R}^{N}(N \geq 1)$ with Lipschitz boundary $\partial \Omega$. We first consider the problem

$$
\begin{cases}\operatorname{div}(a(x, u) \nabla u)=\operatorname{div} b(x, u, \nabla u)+f(x, u) & \text { in } \Omega, \\ u=g & \text { on } \partial \Omega\end{cases}
$$

where $a: \Omega \times \mathbb{R} \rightarrow \mathcal{S}_{N}(\mathbb{R}), b: \Omega \times \mathbb{R} \times \mathbb{R}^{N} \rightarrow \mathbb{R}^{N}, f: \Omega \times \mathbb{R} \rightarrow \mathbb{R}$ are Carathéodory functions (that is, they are measurable in $x \in \Omega$ and continuous in the other variables), $g \in H^{1}(\Omega)$, and $\mathcal{S}_{N}(\mathbb{R})$ denotes the space of $N \times N$ sized symmetric matrices. In the following definition we make clear what we understand by solution to problem (1).

Definition 1 A (weak) solution of problem (1) is an element $u \in H^{1}(\Omega)$ such that $u-g \in$ $H_{0}^{1}(\Omega), a(\cdot, u) \nabla u \in L^{2}(\Omega)^{N}, b(\cdot, u) \in L^{2}(\Omega)^{N}, f(\cdot, u) \in L^{2}(\Omega)$, and

$$
\int_{\Omega}(a(x, u) \nabla u) \cdot \nabla v d x=\int_{\Omega} b(x, u, \nabla u) \cdot \nabla v d x-\int_{\Omega} f(x, u) v d x \quad \text { for all } v \in H_{0}^{1}(\Omega)
$$

Next we focus on nonhomogeneous Dirichlet problems where, contrary to problem (1), the dependence with respect to the gradient $\nabla u$ of the solution $u$ is not expressed in a

C2014 Motreanu and Motreanu; licensee Springer. This is an Open Access article distributed under the terms of the Creative Commons Attribution License (http://creativecommons.org/licenses/by/2.0), which permits unrestricted use, distribution, and reproduction in any medium, provided the original work is properly cited. 
divergence form, namely

$$
\begin{cases}\operatorname{div}(a(x, u) \nabla u)=\operatorname{div} b_{0}(x, u)+\sum_{i=1}^{N} b_{i}(x, u) \frac{\partial u}{\partial x_{i}}+f(x, u) & \text { in } \Omega, \\ u=g & \text { on } \partial \Omega .\end{cases}
$$

Here $a: \Omega \times \mathbb{R} \rightarrow \mathcal{S}_{N}(\mathbb{R}), f: \Omega \times \mathbb{R} \rightarrow \mathbb{R}$ and $g$ are as in problem (1), while $b_{0}: \Omega \times \mathbb{R} \rightarrow \mathbb{R}^{N}$ and $b_{i}: \Omega \times \mathbb{R} \rightarrow \mathbb{R}(i \in\{1, \ldots, N\})$ are Carathéodory functions. The meaning of solution of problem (2) is as follows.

Definition 2 A (weak) solution of problem (2) is an element $u \in H^{1}(\Omega)$ such that $u-$ $g \in H_{0}^{1}(\Omega), a(\cdot, u) \nabla u \in L^{2}(\Omega)^{N}, b_{0}(\cdot, u) \in L^{2}(\Omega)^{N}, b_{i}(\cdot, u) \frac{\partial u}{\partial x_{i}} \in L^{2}(\Omega)$ for all $i \in\{1, \ldots, N\}$, $f(\cdot, u) \in L^{2}(\Omega)$, and

$$
\int_{\Omega}(a(x, u) \nabla u) \cdot \nabla v d x=\int_{\Omega} b_{0}(x, u) \cdot \nabla v d x-\sum_{i=1}^{N} \int_{\Omega} b_{i}(x, u) \frac{\partial u}{\partial x_{i}} v d x-\int_{\Omega} f(x, u) v d x
$$

for all $v \in H_{0}^{1}(\Omega)$.

Problems of type (1) and (2) have been investigated in settings that are different from ours (see, e.g., [1-6]). For instance, problem (1) is studied in [3] when $N=1$ and $N=2$ with functions $a(x, s)$ and $b(x, s, \xi)=b(x, s)$ corresponding to certain physical models, as described by Reynolds equation where $b(x, s)=h(x) \rho(s) V$ with $h(x) \in \mathbb{R}, \rho(s) \geq 0, \rho(0)=0$, and $V \in \mathbb{R}^{N}$. Whereas many of the previous results on problems (1) and (2) involve technical and somewhat restrictive assumptions on the data, the purpose of the present paper is to provide an elementary resolution of problems (1) and (2) in geometrically relevant situation. As an example of such a geometrically relevant situation, we mention the assumption on the term $b(x, \cdot, \xi)$ in problem (1) to vanish at two points.

Our results are stated as Theorems 1 and 2. They are existence and location theorems on problems (1) and (2), respectively, guaranteeing solutions in the sense of Definitions 1 and 2 that fulfill an estimate $\gamma_{*} \leq u \leq \gamma^{*}$ with given constants $\gamma_{*} \leq \gamma^{*}$. This a priori estimate of the solution is derived through natural geometric hypotheses that can be directly checked. It is also worthwhile to remark that we cannot drop by translation the nonhomogeneous boundary conditions to become homogeneous because our hypotheses would be no longer verified. The arguments used in the proof are based on truncation techniques and Schauder's fixed point theorem. We emphasize that, due to the type of assumptions we impose, it is essential in our approach to keep separate the two terms in divergence form appearing in the statement of (1) and (2). A careful inspection of our proofs shows that we rely on the linearity with respect to the gradient $\nabla u$ in the first divergence term and on the vanishing at suitable points in the second divergence term.

The rest of the paper is organized as follows. Section 2 is devoted to problem (1). Section 3 studies problem (2).

\section{Result on problem (1)}

Throughout the paper the notation $\|\cdot\|_{L^{2}}$ and $\|\cdot\|_{H^{1}}$ stands for the usual norms on $L^{2}(\Omega)$ (or $L^{2}\left(\Omega, \mathbb{R}^{N}\right)$ ) and $H^{1}(\Omega)$, respectively. By $|\cdot|$ we denote the Euclidean norm of $\mathbb{R}^{N}$. 
Let $\lambda_{1}$ be the first eigenvalue of the negative Laplacian differential operator on $H_{0}^{1}(\Omega)$, which is known to be positive and characterized by

$$
\lambda_{1}=\inf \left\{\frac{\|\nabla v\|_{L^{2}}^{2}}{\|v\|_{L^{2}}^{2}}: v \in H_{0}^{1}(\Omega), v \neq 0\right\}
$$

We suppose the following hypotheses on the data $a, b, f$, and $g$ in problem (1):

$\left(\mathrm{H}_{1}\right)$ There is a Carathéodory function $\lambda: \Omega \times \mathbb{R} \rightarrow(0,+\infty)$ such that

$$
(a(x, s) \xi) \cdot \xi \geq \lambda(x, s)|\xi|^{2} \quad \text { for a.a. } x \in \Omega \text {, all } s \in \mathbb{R} \text {, all } \xi \in \mathbb{R}^{N} \text {. }
$$

$\left(\mathrm{H}_{2}\right)$ There are constants $\gamma_{*}, \gamma^{*} \in \mathbb{R}$ with $\gamma_{*} \leq g(x) \leq \gamma^{*}$ on $\partial \Omega$ such that

$$
\begin{aligned}
& b\left(x, \gamma_{*}, \xi\right)=b\left(x, \gamma^{*}, \xi\right)=0 \quad \text { for a.a. } x \in \Omega, \text { all } \xi \in \mathbb{R}^{N}, \\
& f\left(x, \gamma_{*}\right)=f\left(x, \gamma^{*}\right)=0 \quad \text { for a.a. } x \in \Omega .
\end{aligned}
$$

$\left(\mathrm{H}_{3}\right)$ The functions $a, f$ are bounded on the set $M:=\left\{(x, s) \in \Omega \times \mathbb{R}: \gamma_{*} \leq s \leq \gamma^{*}\right\}$ and

$$
|b(x, s, \xi)| \leq C(1+|\xi|) \quad \text { for a.a. } x \in \Omega \text {, all } s \in\left[\gamma_{*}, \gamma^{*}\right] \text {, all } \xi \in \mathbb{R}^{N} \text {. }
$$

$\left(\mathrm{H}_{4}\right)$ There is a Carathéodory function $v: \Omega \times \mathbb{R} \rightarrow(0,+\infty)$ such that

$$
\rho:=\inf _{(x, s) \in M}(\lambda(x, s)-v(x, s))>0
$$

and

$$
\begin{aligned}
& (b(x, s, \xi)-b(x, s, \eta)) \cdot(\xi-\eta) \leq v(x, s)|\xi-\eta|^{2} \\
& \quad \text { for a.a. } x \in \Omega \text {, all } s \in \mathbb{R}, \xi, \eta \in \mathbb{R}^{N} .
\end{aligned}
$$

Remark 1 The constants $\gamma_{*}$ and $\gamma^{*}$ are not solutions of problem (1), unless $g \equiv \gamma_{*}$ or $g \equiv \gamma^{*}$ on $\partial \Omega$. Thus, in general, problem (1) has no evident solution.

Remark 2 Due to their different structure and requirements, the two terms in (1) that are in divergence form cannot be combined.

Remark 3 The last part of hypothesis $\left(\mathrm{H}_{4}\right)$ incorporates the monotonicity condition

$$
(b(x, s, \xi)-b(x, s, \eta)) \cdot(\xi-\eta) \leq 0 \quad \text { for a.a. } x \in \Omega \text {, all } s \in \mathbb{R} \text {, all } \xi, \eta \in \mathbb{R}^{N},
$$

as well as the Lipschitz condition

$$
|b(x, s, \xi)-b(x, s, \eta)| \leq v(x, s)|\xi-\eta| \quad \text { for a.a. } x \in \Omega \text {, all } s \in \mathbb{R} \text {, all } \xi, \eta \in \mathbb{R}^{N},
$$

and it is more general than both of them.

The result that we set forth in this section is the following theorem ensuring existence and location of solution for problem (1). 
Theorem 1 Assume that hypotheses $\left(\mathrm{H}_{1}\right)-\left(\mathrm{H}_{4}\right)$ are satisfied. Then problem (1) has at least one solution $u \in H^{1}(\Omega)$ in the sense of Definition 1 satisfying

$$
\gamma_{*} \leq u(x) \leq \gamma^{*} \text { for a.a. } x \in \Omega,
$$

with $\gamma_{*}$ and $\gamma^{*}$ given in $\left(\mathrm{H}_{2}\right)$.

Proof Consider the set

$$
C=\left\{u \in L^{2}(\Omega): \gamma_{*} \leq u(x) \leq \gamma^{*} \text { a.e. } x \in \Omega\right\},
$$

which is a nonempty, bounded, closed, convex subset in $L^{2}(\Omega)$.

Claim 1: Given $u \in C$, there is a unique solution $w_{u} \in H^{1}(\Omega)$ of the problem

$$
\left\{\begin{array}{l}
\int_{\Omega}\left(a(x, u) \nabla w_{u}\right) \cdot \nabla v d x=\int_{\Omega} b\left(x, u, \nabla w_{u}\right) \cdot \nabla v d x-\int_{\Omega} f(x, u) v d x \quad \text { for all } v \in H_{0}^{1}(\Omega) \\
w_{u}-g \in H_{0}^{1}(\Omega)
\end{array}\right.
$$

Note that Claim 1 is equivalent to solving uniquely the problem

$$
\left\{\begin{array}{l}
\langle A(w), v\rangle=B(v) \quad \text { for all } v \in H_{0}^{1}(\Omega) \\
w \in H_{0}^{1}(\Omega)
\end{array}\right.
$$

Here $A: H_{0}^{1}(\Omega) \rightarrow H^{-1}(\Omega)$ and $B \in H^{-1}(\Omega)$ in (4) are expressed by

$$
\langle A(w), v\rangle=\int_{\Omega}(a(x, u) \nabla w-b(x, u, \nabla(w+g))) \cdot \nabla v d x
$$

and

$$
B(v)=-\int_{\Omega}(a(x, u) \nabla g) \cdot \nabla v d x-\int_{\Omega} f(x, u) v d x
$$

for all $w, v \in H_{0}^{1}(\Omega)$. Notice that the operators $A$ and $B$ are well defined due to our hypotheses.

With the fixed element $u \in C$, let us introduce the Carathéodory map $\tilde{a}: \Omega \times \mathbb{R}^{N} \rightarrow \mathbb{R}^{N}$ by

$$
\tilde{a}(x, \xi)=a(x, u(x)) \xi-b(x, u(x), \xi+\nabla g(x)) \quad \text { for a.a. } x \in \Omega \text {, all } \xi \in \mathbb{R}^{N} .
$$

From hypotheses $\left(\mathrm{H}_{1}\right),\left(\mathrm{H}_{3}\right),\left(\mathrm{H}_{4}\right)$, and because $u \in C$, it follows that $\tilde{a}$ satisfies the properties: there is a constant $c_{0}>0$ such that

$$
|\tilde{a}(x, \xi)| \leq c_{0}(1+|\xi|) \quad \text { for a.a. } x \in \Omega \text {, all } \xi \in \mathbb{R}^{N}
$$

and

$$
(\tilde{a}(x, \xi)-\tilde{a}(x, \eta)) \cdot(\xi-\eta) \geq \rho|\xi-\eta|^{2} \quad \text { for a.a. } x \in \Omega \text {, all } \xi, \eta \in \mathbb{R}^{N} .
$$


Estimate (5) guarantees that the operator $A$ is bounded (in the sense to be bounded on bounded sets). It is easily seen that (6) implies that $A$ is coercive, that is,

$$
\lim _{\|\nabla v\|_{L^{2}} \rightarrow+\infty} \frac{\langle A(v), v\rangle}{\|\nabla v\|_{L^{2}}}=+\infty
$$

Moreover, relations (5)-(6) ensure that the operator $A$ is maximal monotone, so pseudomonotone (see, e.g., [7, $\$ 2.3 .1]$ ). Since $A$ is bounded, coercive, and pseudomonotone, it is surjective (see, e.g., [7, Theorem 2.99]), whence the existence of $w_{u}$ in Claim 1. The uniqueness of $w_{u}$ is a direct consequence of (6) (notice that $\rho>0$ ). This establishes Claim 1 .

Now, taking advantage of Claim 1, we define the operator $T: C \rightarrow H^{1}(\Omega)$ by $T(u)=w_{u}$ for all $u \in C$, where $w_{u}$ is the unique element corresponding to $u \in C$ as proved in Claim 1 .

Claim 2: The mapping $T: C \rightarrow H^{1}(\Omega)$ is continuous.

Let $u \in C$ and let $\left\{u_{n}\right\}_{n \geq 1} \subset C$ be a sequence such that $u_{n} \rightarrow u$ in $L^{2}(\Omega)$. Denote $w_{n}=$ $T\left(u_{n}\right)$ and $w=T(u)$. Using the definition of $T$ and choosing $v=w_{n}-w \in H_{0}^{1}(\Omega)$ as a test function in Claim 1 (written with $u_{n}$ and $u$ ), we have

$$
\begin{aligned}
\int_{\Omega} & \left(a\left(x, u_{n}\right) \nabla\left(w-w_{n}\right)\right) \cdot \nabla\left(w-w_{n}\right) d x \\
= & \int_{\Omega}\left(\left(a\left(x, u_{n}\right)-a(x, u)\right) \nabla w\right) \cdot \nabla\left(w-w_{n}\right) d x+\int_{\Omega}(a(x, u) \nabla w) \cdot \nabla\left(w-w_{n}\right) d x \\
& \quad-\int_{\Omega}\left(a\left(x, u_{n}\right) \nabla w_{n}\right) \cdot \nabla\left(w-w_{n}\right) d x \\
= & \int_{\Omega}\left(\left(a\left(x, u_{n}\right)-a(x, u)\right) \nabla w+b(x, u, \nabla w)-b\left(x, u_{n}, \nabla w_{n}\right)\right) \cdot \nabla\left(w-w_{n}\right) d x \\
& \quad-\int_{\Omega}\left(f(x, u)-f\left(x, u_{n}\right)\right)\left(w-w_{n}\right) d x .
\end{aligned}
$$

Combining this formula with $\left(\mathrm{H}_{1}\right),\left(\mathrm{H}_{3}\right),\left(\mathrm{H}_{4}\right),(3)$ and the Cauchy-Schwarz inequality, we obtain

$$
\begin{aligned}
& \int_{\Omega} \lambda\left(x, u_{n}\right)\left|\nabla\left(w-w_{n}\right)\right|^{2} d x \\
& \quad \leq\left\|\left(a\left(x, u_{n}\right)-a(x, u)\right) \nabla w+b(x, u, \nabla w)-b\left(x, u_{n}, \nabla w\right)\right\|_{L^{2}}\left\|\nabla\left(w-w_{n}\right)\right\|_{L^{2}} \\
& \quad+\frac{1}{\sqrt{\lambda_{1}}}\left\|f\left(x, u_{n}\right)-f(x, u)\right\|_{L^{2}}\left\|\nabla\left(w-w_{n}\right)\right\|_{L^{2}}+\int_{\Omega} v\left(x, u_{n}\right)\left|\nabla\left(w-w_{n}\right)\right|^{2} d x .
\end{aligned}
$$

Taking into account hypothesis $\left(\mathrm{H}_{4}\right)$ leads to

$$
\begin{aligned}
\rho\left\|\nabla\left(w-w_{n}\right)\right\|_{L^{2}} \leq & \left\|\left(a\left(x, u_{n}\right)-a(x, u)\right) \nabla w+b(x, u, \nabla w)-b\left(x, u_{n}, \nabla w\right)\right\|_{L^{2}} \\
& +\frac{1}{\sqrt{\lambda_{1}}}\left\|f\left(x, u_{n}\right)-f(x, u)\right\|_{L^{2}} .
\end{aligned}
$$

Set

$$
h_{n}(x)=\left(a\left(x, u_{n}\right)-a(x, u)\right) \nabla w+b(x, u, \nabla w)-b\left(x, u_{n}, \nabla w\right) .
$$

We claim that

$$
h_{n} \rightarrow 0 \quad \text { in } L^{2}(\Omega) .
$$


To this end, we show that any subsequence of $\left\{h_{n}\right\}_{n \geq 1}$ possesses a subsequence converging to 0 in $L^{2}(\Omega)$. Since $u_{n} \rightarrow u$ in $L^{2}(\Omega)$, we have that, along a relabeled subsequence, $h_{n}(x) \rightarrow 0$ for a.a. $x \in \Omega$. Invoking $\left(\mathrm{H}_{3}\right)$, we have that $\left|h_{n}(x)\right|^{2} \leq c_{1}\left(|\nabla w(x)|^{2}+1\right)$, with some constant $c_{1}>0$. Through Lebesgue's dominated convergence theorem, we conclude that $\left\|h_{n}\right\|_{L^{2}} \rightarrow 0$ as $n \rightarrow \infty$, so (8) holds true.

Similarly, we have

$$
f\left(x, u_{n}\right)-f(x, u) \rightarrow 0 \quad \text { in } L^{2}(\Omega) .
$$

Then, in view of (7), we infer that $\left\|\nabla\left(w_{n}-w\right)\right\|_{L^{2}} \rightarrow 0$. Since the domain $\Omega$ is bounded and $w_{n}-w \in H_{0}^{1}(\Omega)$, we can make use of the Poincaré inequality for $w_{n}-w$, which yields $\left\|w_{n}-w\right\|_{L^{2}} \rightarrow 0$, whence $w_{n} \rightarrow w$ in $H^{1}(\Omega)$. This establishes Claim 2 .

With the truncation function $\tau: \mathbb{R} \rightarrow \mathbb{R}$ defined by

$$
\tau(s)= \begin{cases}\gamma_{*} & \text { if } s<\gamma_{*}, \\ s & \text { if } \gamma_{*} \leq s \leq \gamma^{*}, \\ \gamma^{*} & \text { if } s>\gamma^{*},\end{cases}
$$

consider the operator $S: C \rightarrow C$ introduced as follows

$$
S(u)(x)=\tau(T(u)(x)) \quad \text { for a.a. } x \in \Omega .
$$

Note that $S$ takes values in $C \cap H^{1}(\Omega)$.

Claim 3: The mapping $S: C \rightarrow C$ has a fixed point.

Since $T: C \rightarrow H^{1}(\Omega)$ is continuous by Claim 2 (thus a fortiori $T: C \rightarrow L^{2}(\Omega)$ is continuous) and $\tau$ is a bounded continuous function, we infer that $S: C \rightarrow C$ is continuous. We claim that $S: C \rightarrow C$ is a compact operator. To this end, it suffices to check that $S(C)$ is relatively compact in $L^{2}(\Omega)$. Because of the compact embedding of $H^{1}(\Omega)$ in $L^{2}(\Omega)$, it is sufficient to prove that $S(C)$ is bounded in $H^{1}(\Omega)$.

Let $u \in C$ and denote $w=T(u)$. By the definition of $T$ and inserting therein the test function $v=w-g \in H_{0}^{1}(\Omega)$, we see that

$$
\begin{aligned}
& \int_{\Omega}(a(x, u) \nabla(w-g)) \cdot \nabla(w-g) d x \\
& \quad=\int_{\Omega}(-a(x, u) \nabla g+b(x, u, \nabla w)) \cdot \nabla(w-g) d x-\int_{\Omega} f(x, u)(w-g) d x .
\end{aligned}
$$

Then, as in the proof of Claim 2, from assumptions $\left(\mathrm{H}_{1}\right)$ and $\left(\mathrm{H}_{4}\right)$ we obtain that

$$
\begin{aligned}
\int_{\Omega} \lambda(x, u)|\nabla(w-g)|^{2} d x \leq & \|-a(x, u) \nabla g+b(x, u, \nabla g)\|_{L^{2}}\|\nabla(w-g)\|_{L^{2}} \\
& +\frac{1}{\sqrt{\lambda_{1}}}\|f(x, u)\|_{L^{2}}\|\nabla(w-g)\|_{L^{2}}+\int_{\Omega} v(x, u)|\nabla(w-g)|^{2} d x,
\end{aligned}
$$

whence, by $\left(\mathrm{H}_{4}\right)$,

$$
\rho\|\nabla(w-g)\|_{L^{2}} \leq c_{2}
$$

with a constant $c_{2}>0$ independent of $u$. 
Using (11), we derive

$$
\begin{aligned}
\|S(u)\|_{H^{1}}^{2} & =\int_{\Omega}|\tau(w(x))|^{2} d x+\int_{\Omega}|\nabla(\tau \circ w)(x)|^{2} d x \\
& \leq \int_{\Omega}\left(\left|\gamma_{*}\right|+\left|\gamma^{*}\right|\right)^{2} d x+\int_{\left\{\gamma_{*} \leq w \leq \gamma^{*}\right\}}|\nabla w|^{2} d x \\
& \leq c_{3}\left(1+\|\nabla(w-g)\|_{L^{2}}^{2}\right) \leq c_{4}
\end{aligned}
$$

with constants $c_{3}, c_{4}>0$ independent of $u$. It follows that the set $S(C)$ is bounded in $H^{1}(\Omega)$, so according to what was said before, the map $S: C \rightarrow C$ is compact. Consequently, Schauder's fixed point theorem can be applied (see, e.g., [8, p.452]), through which it follows that $S$ admits a fixed point in $C$. This shows Claim 3.

Claim 4: Let $u \in C$ be a fixed point of $S$. Then there holds $T(u)=u$.

The existence of a point $u \in C$ such that $S(u)=u$ is ensured by Claim 3. Fix such a point $u$ and set $w=T(u)$. In order to deduce the desired conclusion from $S(u)=u$, it suffices to check that $\gamma_{*} \leq w \leq \gamma^{*}$ a.e. in $\Omega$. We only verify the inequality $\gamma_{*} \leq w$ a.e. in $\Omega$ because the proof of the other inequality is similar. By virtue of hypothesis $\left(\mathrm{H}_{2}\right)$, we have $w=g \geq \gamma_{*}$ on $\partial \Omega$ (in the sense of traces), hence $\left(w-\gamma_{*}\right)^{-}=0$ on $\partial \Omega$ and so the function $\left(w-\gamma_{*}\right)^{-}$ belongs to $H_{0}^{1}(\Omega)$ (see, e.g., [7, p.35]). Using $v=\left(w-\gamma_{*}\right)^{-}$as a test function in the definition of $T$ gives

$$
\begin{aligned}
\int_{\Omega}(a(x, u) \nabla w) \cdot \nabla\left(w-\gamma_{*}\right)^{-} d x= & \int_{\Omega} b(x, u, \nabla w) \cdot \nabla\left(w-\gamma_{*}\right)^{-} d x \\
& -\int_{\Omega} f(x, u)\left(w-\gamma_{*}\right)^{-} d x,
\end{aligned}
$$

which reads as

$$
\begin{aligned}
\int_{\left\{\gamma_{*} \geq w\right\}}(a(x, u) \nabla w) \cdot \nabla w d x= & \int_{\left\{\gamma_{*} \geq w\right\}} b(x, u, \nabla w) \cdot \nabla w d x \\
& -\int_{\left\{\gamma_{*} \geq w\right\}} f(x, u)\left(w-\gamma_{*}\right) d x
\end{aligned}
$$

By the assumption that $S(u)=u$ and from (10) we know that $u(x)=S(u)(x)=\tau(w(x))$ for a.a. $x \in \Omega$, hence $u=\gamma_{*}$ a.e. in $\left\{\gamma_{*} \geq w\right\}$. Then hypothesis $\left(\mathrm{H}_{2}\right)$ implies that $b(x, u, \nabla w)=0$ and $f(x, u)=0$ a.e. in $\left\{\gamma_{*} \geq w\right\}$. Consequently, (12), $\left(\mathrm{H}_{1}\right)$, and $\left(\mathrm{H}_{4}\right)$ entail

$$
\int_{\left\{\gamma_{*} \geq w\right\}}|\nabla w|^{2} d x \leq 0
$$

whence $\nabla\left(w-\gamma_{*}\right)^{-}=-\nabla w=0$ a.e. in $\left\{\gamma_{*} \geq w\right\}$. On the other hand, we have $\nabla\left(w-\gamma_{*}\right)^{-}=0$ in $\left\{\gamma_{*}<w\right\}$. Altogether, we obtain that $\nabla\left(w-\gamma_{*}\right)^{-}=0$ in $\Omega$. Since $\left(w-\gamma_{*}\right)^{-} \in H_{0}^{1}(\Omega)$, we conclude that $\left(w-\gamma_{*}\right)^{-}=0$ a.e. in $\Omega$, thus $w \geq \gamma_{*}$ a.e. in $\Omega$. This proves Claim 4 .

By Claims 3 and 4, the operator $T$ admits a fixed point $u \in C$. Then the definition of $T$ implies that $u=T(u) \in g+H_{0}^{1}(\Omega)$, so $u$ is a solution of problem (1). In addition, the fact that $u \in C$ guarantees that $\gamma_{*} \leq u \leq \gamma^{*}$ a.e. in $\Omega$. The proof of Theorem 1 is complete. 


\section{Result on problem (2)}

The hypotheses on the data $a, b_{i}(i \in\{0,1, \ldots, N\}), f$, and $g$ in problem (2) that we suppose are as follows: $\left(\mathrm{H}_{1}\right)$ in Section 2,

$\left(\mathrm{H}_{2}^{\prime}\right)$ There exist constants $\gamma_{*}, \gamma^{*} \in \mathbb{R}$ such that $\gamma_{*} \leq g(x) \leq \gamma^{*}$ on $\partial \Omega$ and

$$
\begin{aligned}
& b_{i}\left(x, \gamma_{*}\right)=b_{i}\left(x, \gamma^{*}\right)=0 \quad \text { for a.a. } x \in \Omega, \text { all } i \in\{0,1, \ldots, N\}, \\
& f\left(x, \gamma_{*}\right)=f\left(x, \gamma^{*}\right)=0 \quad \text { for a.a. } x \in \Omega .
\end{aligned}
$$

$\left(\mathrm{H}_{3}^{\prime}\right)$ There exist constants $m>0$ and $\tilde{m}, m_{0} \geq 0$ such that

$$
\begin{aligned}
& |a(x, s)| \leq m, \quad|f(x, s)| \leq \tilde{m}, \quad \text { and } \quad\left|b_{0}(x, s)\right| \leq m_{0} \\
& \text { for a.a. } x \in \Omega \text {, all } s \in\left[\gamma_{*}, \gamma^{*}\right] .
\end{aligned}
$$

$\left(\mathrm{H}_{4}^{\prime}\right) \mu:=\inf _{(x, s) \in \Omega \times\left[\gamma_{*}, \gamma^{*}\right]} \lambda(x, s)>0$ and there exist constants $m_{i} \geq 0, i \in\{1, \ldots, N\}$, with $\bar{m}:=\sqrt{\sum_{i=1}^{N} m_{i}^{2}}<\mu \sqrt{\lambda_{1}}$, such that

$$
\left|b_{i}(x, s)\right| \leq m_{i} \quad \text { for a.a. } x \in \Omega \text {, all } s \in\left[\gamma_{*}, \gamma^{*}\right], i \in\{1, \ldots, N\} .
$$

Remark 4 As in the case of problem (1), we note that the constant functions $u \equiv \gamma_{*}$ and $u \equiv \gamma^{*}$ are not solutions of problem (2), unless $g \equiv \gamma_{*}$ or $g \equiv \gamma^{*}$ on $\partial \Omega$.

Now we state our result of existence and location of solutions for problem (2).

Theorem 2 Assume that $\left(\mathrm{H}_{1}\right),\left(\mathrm{H}_{2}^{\prime}\right),\left(\mathrm{H}_{3}^{\prime}\right)$, and $\left(\mathrm{H}_{4}^{\prime}\right)$ are satisfied. Then problem (2) has at least one solution $u \in H^{1}(\Omega)$ in the sense of Definition 2 satisfying

$$
\gamma_{*} \leq u(x) \leq \gamma^{*} \quad \text { for a.a. } x \in \Omega,
$$

with $\gamma_{*}$ and $\gamma^{*}$ as in $\left(\mathrm{H}_{2}^{\prime}\right)$.

Proof We follow the pattern of proof of Theorem 1. Hence, using the constants $\gamma_{*}$ and $\gamma^{*}$ prescribed in $\left(\mathrm{H}_{2}^{\prime}\right)$, we consider

$$
C=\left\{u \in L^{2}(\Omega): \gamma_{*} \leq u(x) \leq \gamma^{*} \text { for a.a. } x \in \Omega\right\}
$$

which is a nonempty, bounded, closed, convex subset of $L^{2}(\Omega)$. We proceed by proving four claims regarding problem (2) that correspond to those in the proof of Theorem 1 for problem (1). We provide the proof since there are some differences with respect to the proof of Theorem 1.

Claim 1: For every $u \in C$, there is a unique solution $w_{u} \in H^{1}(\Omega)$ of the problem

$$
\left\{\begin{array}{l}
\int_{\Omega}\left(a(x, u) \nabla w_{u}\right) \cdot \nabla v d x \\
\quad=\int_{\Omega} b_{0}(x, u) \cdot \nabla v d x-\sum_{i=1}^{N} \int_{\Omega} b_{i}(x, u) \frac{\partial w_{u}}{\partial x_{i}} v d x-\int_{\Omega} f(x, u) v d x \quad \text { for all } v \in H_{0}^{1}(\Omega) \\
w_{u}-g \in H_{0}^{1}(\Omega)
\end{array}\right.
$$


As in the proof of Theorem 1, first we note that Claim 1 is equivalent to proving that the problem

$$
\left\{\begin{array}{l}
A(w, v)=B(v) \quad \text { for all } v \in H_{0}^{1}(\Omega) \\
w \in H_{0}^{1}(\Omega)
\end{array}\right.
$$

admits a unique solution, where

$$
A(w, v)=\int_{\Omega}(a(x, u) \nabla w) \cdot \nabla v d x+\sum_{i=1}^{N} \int_{\Omega} b_{i}(x, u) \frac{\partial w}{\partial x_{i}} v d x
$$

and

$$
B(v)=\int_{\Omega}\left(b_{0}(x, u)-a(x, u) \nabla g\right) \cdot \nabla v d x-\sum_{i=1}^{N} \int_{\Omega} b_{i}(x, u) \frac{\partial g}{\partial x_{i}} v d x-\int_{\Omega} f(x, u) v d x .
$$

For $u \in C$, by the Cauchy-Schwarz inequalities in $L^{2}(\Omega)$ and in $\mathbb{R}^{N}$, as well as $\left(\mathrm{H}_{4}^{\prime}\right)$ and (3), we derive the estimate

$$
\begin{aligned}
\left|\sum_{i=1}^{N} \int_{\Omega} b_{i}(x, u) \frac{\partial w}{\partial x_{i}} v d x\right| & \leq \sum_{i=1}^{N} \int_{\Omega}\left|b_{i}(x, u) \frac{\partial w}{\partial x_{i}}\right||v| d x \\
& \leq\|v\|_{L^{2}} \sum_{i=1}^{N}\left(\int_{\Omega}\left|b_{i}(x, u)\right|^{2}\left|\frac{\partial w}{\partial x_{i}}\right|^{2} d x\right)^{\frac{1}{2}} \\
& \leq\|v\|_{L^{2}} \sum_{i=1}^{N} m_{i}\left(\int_{\Omega}\left|\frac{\partial w}{\partial x_{i}}\right|^{2} d x\right)^{\frac{1}{2}} \\
& \leq\|v\|_{L^{2}}\left(\sum_{i=1}^{N} m_{i}^{2}\right)^{\frac{1}{2}}\left(\sum_{i=1}^{N} \int_{\Omega}\left|\frac{\partial w}{\partial x_{i}}\right|^{2} d x\right)^{\frac{1}{2}} \\
& =\|v\|_{L^{2}} \bar{m}\left(\int_{\Omega}|\nabla w|^{2} d x\right)^{\frac{1}{2}} \\
& \leq \frac{\bar{m}}{\sqrt{\lambda_{1}}}\|\nabla v\|_{L^{2}}\|\nabla w\|_{L^{2}}
\end{aligned}
$$

for all $w, v \in H_{0}^{1}(\Omega)$. Using the Cauchy-Schwarz inequality in $L^{2}(\Omega)$, the fact that $u \in C$, $\left(\mathrm{H}_{3}^{\prime}\right)$ and $(14)$, we get

$$
\begin{aligned}
|A(w, v)| & \leq\|a(x, u) \nabla w\|_{L^{2}}\|\nabla v\|_{L^{2}}+\left|\sum_{i=1}^{N} \int_{\Omega} b_{i}(x, u) \frac{\partial w}{\partial x_{i}} v d x\right| \\
& \leq\left(m+\frac{\bar{m}}{\sqrt{\lambda_{1}}}\right)\|\nabla w\|_{L^{2}}\|\nabla v\|_{L^{2}} \quad \text { for all } w, v \in H_{0}^{1}(\Omega),
\end{aligned}
$$

which ensures that $A: H_{0}^{1}(\Omega) \times H_{0}^{1}(\Omega) \rightarrow \mathbb{R}$ is a continuous bilinear form. From $\left(\mathrm{H}_{1}\right)$, the fact that $u \in C,\left(\mathrm{H}_{4}^{\prime}\right)$ and (14), we have

$$
A(v, v) \geq\left(\mu-\frac{\bar{m}}{\sqrt{\lambda_{1}}}\right)\|\nabla v\|_{L^{2}}^{2} \quad \text { for all } v \in H_{0}^{1}(\Omega)
$$


Since $\bar{m}<\mu \sqrt{\lambda_{1}}$ (as postulated in $\left(\mathrm{H}_{4}^{\prime}\right)$ ), we infer that $A: H_{0}^{1}(\Omega) \times H_{0}^{1}(\Omega) \rightarrow \mathbb{R}$ is also coercive.

On the basis of the reasoning in (14), the following estimate holds

$$
\left|\sum_{i=1}^{N} \int_{\Omega} b_{i}(x, u) \frac{\partial g}{\partial x_{i}} v d x\right| \leq \frac{\bar{m}}{\sqrt{\lambda_{1}}}\|\nabla g\|_{L^{2}}\|\nabla v\|_{L^{2}}
$$

for all $v \in H_{0}^{1}(\Omega)$. Taking into account that $u \in C,\left(\mathrm{H}_{3}^{\prime}\right),(15)$ and (3), we see that

$$
|B(v)| \leq\left(\left(m+\frac{\bar{m}}{\sqrt{\lambda_{1}}}\right)\|\nabla g\|_{L^{2}}+\left(m_{0}+\frac{\tilde{m}}{\sqrt{\lambda_{1}}}\right)|\Omega|^{\frac{1}{2}}\right)\|\nabla v\|_{L^{2}} \quad \text { for all } v \in H_{0}^{1}(\Omega)
$$

where $|\Omega|$ stands for the Lebesgue measure of $\Omega$. Therefore $B: H_{0}^{1}(\Omega) \rightarrow \mathbb{R}$ is linear and continuous. The properties of the mappings $A$ and $B$ permit to apply the Lax-Milgram theorem, through which we conclude that problem (13) admits a unique solution. This establishes Claim 1.

As in the proof of Theorem 1, we introduce the operator $T: C \rightarrow H^{1}(\Omega)$ defined by $T(u)=w_{u}$ for all $u \in C$, with $w_{u}$ given in Claim 1 .

Claim 2: The mapping $T: C \rightarrow H^{1}(\Omega)$ is continuous.

In order to prove this assertion, we proceed as in the proof of Claim 2 in Theorem 1. Fix $u \in C$ and consider a sequence $\left\{u_{n}\right\}_{n \geq 1} \subset C$ such that $u_{n} \rightarrow u$ in $L^{2}(\Omega)$. Denoting $w_{n}=$ $T\left(u_{n}\right)$ and $w=T(u)$, we find that

$$
\begin{aligned}
\int_{\Omega}\left(a\left(x, u_{n}\right) \nabla\left(w-w_{n}\right)\right) \cdot \nabla\left(w-w_{n}\right) d x \\
=\int_{\Omega}\left(\left(a\left(x, u_{n}\right)-a(x, u)\right) \nabla w+b_{0}(x, u)-b_{0}\left(x, u_{n}\right)\right) \cdot \nabla\left(w-w_{n}\right) d x \\
\quad-\sum_{i=1}^{N} \int_{\Omega}\left[\left(b_{i}(x, u)-b_{i}\left(x, u_{n}\right)\right) \frac{\partial w}{\partial x_{i}}+b_{i}\left(x, u_{n}\right) \frac{\partial\left(w-w_{n}\right)}{\partial x_{i}}\right]\left(w-w_{n}\right) d x \\
\quad-\int_{\Omega}\left(f(x, u)-f\left(x, u_{n}\right)\right)\left(w-w_{n}\right) d x .
\end{aligned}
$$

A straightforward calculation entails

$$
\left|\sum_{i=1}^{N} \int_{\Omega} b_{i}\left(x, u_{n}\right) \frac{\partial\left(w-w_{n}\right)}{\partial x_{i}}\left(w-w_{n}\right) d x\right| \leq \frac{\bar{m}}{\sqrt{\lambda_{1}}}\left\|\nabla\left(w-w_{n}\right)\right\|_{L^{2}}^{2} .
$$

Combining $\left(\mathrm{H}_{1}\right),\left(\mathrm{H}_{3}^{\prime}\right),\left(\mathrm{H}_{4}^{\prime}\right)$, (16), (17), (3), and the Cauchy-Schwarz inequality yields

$$
\begin{aligned}
\mu\left\|\nabla\left(w-w_{n}\right)\right\|_{L^{2}} \leq & \left\|\left(a\left(x, u_{n}\right)-a(x, u)\right) \nabla w+b_{0}(x, u)-b_{0}\left(x, u_{n}\right)\right\|_{L^{2}} \\
& +\frac{1}{\sqrt{\lambda_{1}}}\left\|\sum_{i=1}^{N}\left(b_{i}(x, u)-b_{i}\left(x, u_{n}\right)\right) \frac{\partial w}{\partial x_{i}}\right\|_{L^{2}} \\
& +\frac{1}{\sqrt{\lambda_{1}}}\left\|f(x, u)-f\left(x, u_{n}\right)\right\|_{L^{2}}+\frac{\bar{m}}{\sqrt{\lambda_{1}}}\left\|\nabla\left(w-w_{n}\right)\right\|_{L^{2}},
\end{aligned}
$$

with $\mu$ and $\bar{m}$ in $\left(\mathrm{H}_{4}^{\prime}\right)$. 
Proceeding as in (8) we show that

$$
\begin{aligned}
& \left(a\left(x, u_{n}\right)-a(x, u)\right) \nabla w+b_{0}(x, u)-b_{0}\left(x, u_{n}\right) \rightarrow 0 \quad \text { in } L^{2}(\Omega), \\
& \sum_{i=1}^{N}\left(b_{i}(x, u)-b_{i}\left(x, u_{n}\right)\right) \frac{\partial w}{\partial x_{i}} \rightarrow 0 \quad \text { and } f(x, u)-f\left(x, u_{n}\right) \rightarrow 0 \quad \text { in } L^{2}(\Omega) .
\end{aligned}
$$

Now it suffices to combine (18), (19), (20) and recall that $\bar{m}<\mu \sqrt{\lambda_{1}}$ (see $\left(\mathrm{H}_{4}^{\prime}\right)$ ) to conclude that $\left\|\nabla\left(w_{n}-w\right)\right\|_{L^{2}} \rightarrow 0$. Then, because $w_{n}-w \in H_{0}^{1}(\Omega)$ and $\Omega$ is bounded, by the Poincaré inequality, we also deduce that $\left\|w_{n}-w\right\|_{L^{2}} \rightarrow 0$. This amounts to saying that $w_{n} \rightarrow w$ in $H^{1}(\Omega)$, which proves Claim 2 .

Following the approach developed in the proof of Theorem 1 , we introduce the operator $S: C \rightarrow C$ given by (10), with the truncation function $\tau: \mathbb{R} \rightarrow \mathbb{R}$ defined in (9) corresponding to the constants $\gamma_{*}$ and $\gamma^{*}$ in $\left(\mathrm{H}_{2}^{\prime}\right)$.

Claim 3: The mapping $S: C \rightarrow C$ has a fixed point.

Claim 2 readily implies that the mapping $S: C \rightarrow C$ is continuous. Let us check that $S: C \rightarrow C$ is a compact operator. To see this, it suffices to check that $S(C)$ is relatively compact in $L^{2}(\Omega)$. Thanks to the compactness of the embedding of $H^{1}(\Omega)$ into $L^{2}(\Omega)$, this reduces to show that $S(C)$ is bounded in $H^{1}(\Omega)$. To this end, let $u \in C$ and denote $w=T(u)$. We can argue as in the proof of Theorem 1 by relying now on the present hypotheses. We obtain from $w=T(u)$ with the test function $v=w-g \in H_{0}^{1}(\Omega)$, in conjunction with $\left(\mathrm{H}_{1}\right)$, $\left(\mathrm{H}_{3}^{\prime}\right),\left(\mathrm{H}_{4}^{\prime}\right)$, that

$$
\begin{aligned}
\mu\|\nabla(w-g)\|_{L^{2}} & \leq\left\|b_{0}(x, u)-a(x, u) \nabla g\right\|_{L^{2}}+\frac{1}{\sqrt{\lambda_{1}}}\left(\bar{m}\|\nabla w\|_{L^{2}}+\tilde{m}\right) \\
& \leq \frac{\bar{m}}{\sqrt{\lambda_{1}}}\|\nabla(w-g)\|_{L^{2}}+c_{0},
\end{aligned}
$$

where $c_{0}>0$ is a constant independent of $u$. In view of hypothesis $\left(\mathrm{H}_{4}^{\prime}\right)$, it follows that

$$
\|\nabla w\|_{L^{2}} \leq c_{1}
$$

with a constant $c_{1}>0$ independent of $u$. Using (21) and the definition of $S$, we get the estimate

$$
\begin{aligned}
\|S(u)\|_{H^{1}}^{2} & =\int_{\Omega}|\tau(w(x))|^{2} d x+\int_{\Omega}|\nabla(\tau \circ w)(x)|^{2} d x \\
& \leq \int_{\Omega}\left(\left|\gamma_{*}\right|+\left|\gamma^{*}\right|\right)^{2} d x+\int_{\left\{\gamma_{*} \leq w \leq \gamma^{*}\right\}}|\nabla w|^{2} d x \leq c_{2},
\end{aligned}
$$

with $c_{2}>0$ independent of $u$. We conclude that the set $S(C)$ is bounded in $H^{1}(\Omega)$, so relatively compact in $L^{2}(\Omega)$. Therefore the map $S: C \rightarrow C$ is compact. This enables us to apply Schauder's fixed point theorem (see, e.g., [8, p.452]), which implies that $S$ possesses a fixed point in C. Claim 3 is thus shown.

Claim 4: If $u \in C$ is a fixed point of $S$, then $T(u)=u$.

Let $u \in C$ be a fixed point of $S$ and set $w=T(u)$. In order to show that $u$ is a fixed point of $T$, it is needed to be fulfilled $\gamma_{*} \leq w \leq \gamma^{*}$ a.e. in $\Omega$. The proof is done following the 
pattern of the corresponding part in the proof of Theorem 1. We outline the proof of $w \geq \gamma_{*}$ a.e. in $\Omega$ (the proof of the other inequality is similar).

Testing in $w=T(u)$ with $v=\left(w-\gamma_{*}\right)^{-} \in H_{0}^{1}(\Omega)$ yields

$$
\begin{aligned}
\int_{\left\{\gamma_{*} \geq w\right\}}(a(x, u) \nabla w) \cdot \nabla w d x= & \int_{\left\{\gamma_{*} \geq w\right\}} b_{0}(x, u) \cdot \nabla w d x \\
& -\int_{\left\{\gamma_{*} \geq w\right\}}\left(\sum_{i=1}^{N} b_{i}(x, u) \frac{\partial w}{\partial x_{i}}+f(x, u)\right)\left(w-\gamma_{*}\right) d x .
\end{aligned}
$$

In $\left\{\gamma_{*} \geq w\right\}$ it is true that

$$
u(x)=S(u)(x)=\tau(w(x))=\gamma_{*} \quad \text { for a.e. } x \in \Omega .
$$

Then hypothesis $\left(\mathrm{H}_{2}^{\prime}\right)$ implies that

$$
b_{i}(x, u)=f(x, u)=0 \quad \text { a.e. in }\left\{\gamma_{*} \geq w\right\}, i \in\{0,1, \ldots, N\} .
$$

Combining with (22), $\left(\mathrm{H}_{1}\right)$, and $\left(\mathrm{H}_{4}^{\prime}\right)$ entails

$$
\int_{\left\{\gamma_{*} \geq w\right\}}|\nabla w|^{2} d x \leq 0
$$

It turns out that $\nabla\left(w-\gamma_{*}\right)^{-}=-\nabla w=0$ a.e. in $\left\{\gamma_{*} \geq w\right\}$. Also, it is clear that $\nabla\left(w-\gamma_{*}\right)^{-}=$ 0 in $\left\{\gamma_{*}<w\right\}$. Consequently, the equality $\nabla\left(w-\gamma_{*}\right)^{-}=0$ in $\Omega$ is valid, which results in $\left(w-\gamma_{*}\right)^{-}=0$ a.e. in $\Omega$ because $\left(w-\gamma_{*}\right)^{-} \in H_{0}^{1}(\Omega)$. This reads as $w \geq \gamma_{*}$ a.e. in $\Omega$, so Claim 4 is fulfilled.

Now we can conclude the proof. Claims 3 and 4 ensure that there exists a fixed point $u \in C$ of the operator $T$. This means that $u=T(u) \in g+H_{0}^{1}(\Omega)$ and $u$ is a solution of problem (2). Moreover, since $u \in C$, we also have $\gamma_{*} \leq u \leq \gamma^{*}$ a.e. in $\Omega$. The desired conclusion is achieved.

Competing interests

The authors declare that they have no competing interests.

Authors' contributions

DM and WM jointly worked and obtained all the results presented in the paper and participated equally in the preparation of the paper. Both authors read and approved the final manuscript.

\section{Author details}

'Département de Mathématiques, Université de Perpignan, Perpignan, 66860, France. ²Department of Mathematics, Ben Gurion University of the Negev, Be'er Sheva, 84105, Israel.

\section{Acknowledgements}

The second author is supported by the Marie Curie Intra-European Fellowship for Career Development within the European Community's 7th Framework Program (Grant Agreement No. PIEF-GA-2010-274519).

Received: 22 October 2013 Accepted: 6 December 2013 Published: 07 Jan 2014

\section{References}

1. Bensoussan, A, Boccardo, L, Murat, F: On a nonlinear partial differential equation having natural growth terms and unbounded solution. Ann. Inst. Henri Poincaré, Anal. Non Linéaire 5, 347-364 (1988)

2. Boccardo, L, Giachetti, D, Diaz, Jl, Murat, F: Existence and regularity of renormalized solutions for some elliptic problems involving derivatives of nonlinear terms. J. Differ. Equ. 106, 215-237 (1993) 
3. Buscaglia, G, Ciuperca, I, Jai, M: Existence and uniqueness for several non-linear elliptic problems arising in lubrication theory. J. Differ. Equ. 218, 187-215 (2005)

4. Ferone, V, Murat, F: Nonlinear problems having natural growth in the gradient: an existence result when the source terms are small. Nonlinear Anal. 42, 1309-1326 (2000)

5. Gilbarg, D, Trudinger, NS: Elliptic Partial Differential Equations of Second Order. Springer, Berlin (2001)

6. Ladyzhenskaya, OA, Ural'tseva, NN: Linear and Quasilinear Elliptic Equations. Academic Press, New York (1968)

7. Carl, S, Le, VK, Motreanu, D: Nonsmooth Variational Problems and Their Inequalities. Comparison Principles and Applications. Springer, New York (2007)

8. Zeidler, E: Nonlinear Functional Analysis and Its Applications. I. Fixed-Point Theorems. Springer, New York (1986)

10.1186/1687-2770-2014-6

Cite this article as: Motreanu and Motreanu: Elliptic problems with nonhomogeneous boundary condition and derivatives of nonlinear terms. Boundary Value Problems 2014, 2014:6

\section{Submit your manuscript to a SpringerOpen ${ }^{0}$ journal and benefit from:}

- Convenient online submission

- Rigorous peer review

- Immediate publication on acceptance

- Open access: articles freely available online

- High visibility within the field

- Retaining the copyright to your article 